Article

\title{
Synthesis and Biological Evaluation of New
} Substituted Hantzsch Thiazole Derivatives from Environmentally Benign One-Pot Synthesis Using Silica Supported Tungstosilisic Acid as Reusable Catalyst

\author{
Houria Bouherrou ${ }^{1}$, Aicha Saidoun ${ }^{1}$, Ahmed Abderrahmani ${ }^{2}$, Lamia Abdellaziz ${ }^{2}$, \\ Yahia Rachedi ${ }^{1, *}$, Françoise Dumas ${ }^{3}$ and Albert Demenceau ${ }^{4}$ \\ 1 Laboratory of Applied Organic Chemistry, Faculty of Chemistry, University of Science and Technology \\ Houari Boumediene, BP 32, El Alia, 16111 Bab Ezzouar, Algiers, Algeria; houriab87@yahoo.fr (H.B.); \\ aicha.saidoun@hotmail.com (A.S.) \\ 2 Laboratory of Cellular and Molecular Biology, Faculty of Biological Sciences, USTHB, BP 32, El Alia, \\ 16111 Bab Ezzouar, Algiers, Algeria; abderrahmaniahmed1@gmail.com (A.A.); \\ abdelaziz.lm@hotmail.com (L.A.) \\ 3 Laboratoire BioCIS, UMR CNRS8076, Chimie des Substances Naturelles, IPSIT and LabEx LERMIT, CNRS, \\ Université Paris-Saclay, Université Paris-Sud, Faculté de Pharmacie, 5, rue Jean-Baptiste Clément, 92296 \\ Châtenay-Malabry CEDEX, France; fran_dumas@yahoo.com \\ 4 Laboratoire de chimie macromoléculaire et catalyse organique, Institut de Chimie (B6a), Université de Liège, \\ Quartier Agora, Allée du 6 Août, 4000 Liège (Sart-Tilman), Belgique; albertdemanceau@gmail.com \\ * Correspondence: hbouherrou@gmail.com; Tel./Fax: +213-2124-7311
}

Academic Editors: Diego Muñoz-Torrero, Rodolfo Lavilla and Christopher Hulme Received: 10 March 2017; Accepted: 4 May 2017; Published: 7 May 2017

\begin{abstract}
An efficient and green method has been developed for the synthesis of new substituted Hantzsch thiazole derivatives in $79 \%-90 \%$ yield, via the one-pot multi-component procedure, by the reaction of 3-(bromoacetyl)-4-hydroxy-6-methyl-2H-pyran-2-one, thiourea and substituted benzaldehydes in the presence of silica supported tungstosilisic acid, as a reusable catalyst, under conventional heating or under ultrasonic irradiation. The catalyst is recoverable by a simple filtration and can be reused in the subsequent reactions. Most of the thiazoles exhibited significant antibacterial activity compared toamoxicillin and ciprofloxacin as positive controls. In addition, the new compounds showed moderate to good antioxidant (DPPH) radical scavenging activity.
\end{abstract}

Keywords: thiazoles; multi-component reaction; Hantzsch condensation; heterogeneous catalysis; antioxidant; antibacterial

\section{Introduction}

Molecular entities bearing thiazole ring system(s) form an important class of natural and synthetic compounds because of their physicochemical properties [1-4].Furthermore, they exhibit a wide range of biological activities such as cardiotonic [5], antifungal [6,7], analgesic [8], anticonvulsant [9], antituberculosis [10], antiviral [11], anti-inflammatory [12], anti-HIV [13,14], and anticancer activities [15,16].

It is known that thiazoles can be synthesized from $\alpha$-bromoketone and a thiourea via Hantzsch thiazole synthesis in high yields [17]. A literature survey revealed that there are numerous routes reported for the synthesis of substituted thiazoles according to Hantzsch thiazole synthesis $[18,19]$. 
Due to the importance of these heterocycles in medicinal and material chemistry, the development of new routes-which leads to these heterocycles in higher yields, shorter reaction time, milder or greener conditions-has received considerable attention in organic synthesis [20-27]. When designing organic synthesis, chemists have to face the ecocompatibility concern of their synthesis plan. Multi-component reactions (MCRs) are a special type of synthetically useful organic reaction, in which, three or more different starting materials react, to generate a single product in a one-pot procedure [28-32]. Thus, MCRs also represent a possible instrument to perform near ideal synthesis, because they possess one of the aforementioned qualities, namely the possibility of building up complex molecules with maximum simplicity and brevity $[33,34]$. On the other hand, the use of catalysis in organic synthesis has received considerable attention owing to its important advantages: it helps to reduce energy consumptionand increases control of selectivity [35-37].In particular, heterogeneous catalysis appears as the solution of choice since many groups have clearly shown that the molecular structure and/or texture of catalytic materials could directly affect the selectivity of the reactions involved [38,39]. Heteropolyacids (HPAs) are widely used as heterogeneous catalysts and offer many advantages, especially polyoxometalate compounds such as tungstosilisic acid $\mathrm{H}_{4} \mathrm{SiW}_{12} \mathrm{O}_{40}$ (SiW) $[36,37]$.

Despite its unusual activation mode, ultrasonic irradiation (US) has been increasingly used in organic synthesis in the last three decades [40,41]. A large number of organic reactions can be carried out in higher yields, shorter reaction time and milder conditions under ultrasound irradiation [42,43]. In comparison to conventional methods, the US method is more convenient and easily controlled [44-46]. Owing to the above facts and in continuation of our research work on the development of MCRs for the synthesis of novel heterocyclic compounds, in this article, we report on the synthesis of functionalized thiazoles, in good yields, using $\mathrm{SiW} . \mathrm{SiO}_{2}$ as a reusable solid support catalyst (Scheme 1) and their biological evaluation.

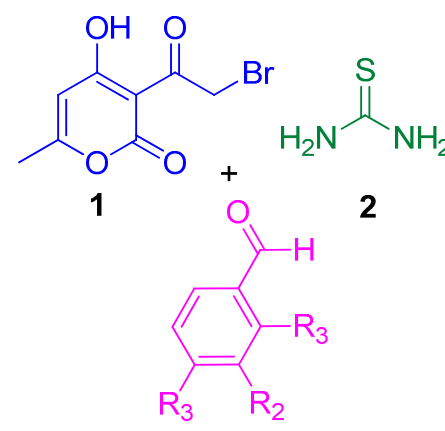

3a-3j

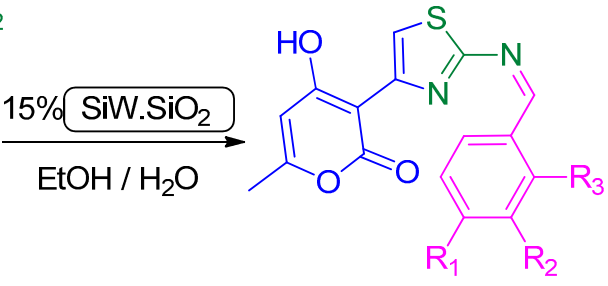

$4 a-4 j$

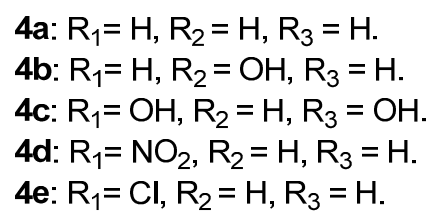

4f: $\mathrm{R}_{1}=\mathrm{OH}, \mathrm{R}_{2}=\mathrm{H}, \mathrm{R}_{3}=\mathrm{H}$.

4g: $\mathrm{R}_{1}=\mathrm{H}, \mathrm{R}_{2}=\mathrm{OH}, \mathrm{R}_{3}=\mathrm{OH}$.

4h: $R_{1}=H, R_{2}=H, R_{3}=\mathrm{OCH}_{3}$.

4i: $R_{1}=O_{3} H_{3}, R_{2}=H, R_{3}=H$.

4j: $R_{1}=H, R_{2}=\mathrm{OCH}_{3}, \mathrm{R}_{3}=\mathrm{H}$.

Scheme 1. $\mathrm{SiW} \cdot \mathrm{SiO}_{2}$ catalyzed synthesis of new Hantzsch thiazole derivatives $\mathbf{4 a - 4} \mathbf{j}$.

\section{Results and Discussion}

\subsection{Chemistry}

The required $\alpha$-haloketone (1), 3-(bromoacetyl)-4-hydroxy-6-methyl-2H-pyran-2-one, is not commercially available. It could be easily obtained via the selective $\alpha$-monobromination of dehydroacetic acid (DHAA) (5) in 70\% yield according to a known procedure [47]. 
In that way, we decided to study the reactivity of (1) with several binucleophilic amines and initiate our studies with the reaction of thiourea (2) to afford the corresponding thiazoles. The thiazoles were assembled according to a slightly modified version of the Hantzsch thiazoles synthesis [48,49]. Herein, the three-component one-pot condensation of an equimolar amount of (1), (2) and substituted benzaldehydes (3a-3j), using $\mathrm{SiW}_{\mathbf{S i O}}$ as a catalyst under conventional heating and under ultrasonic irradiation, yielded the corresponding thiazole derivatives (4a-4j) in good to excellent yield. In order to enhance the process, the reaction condition was optimized for the transformation of (1), (2) and benzaldehyde (3a) under different conditions of temperature, solvents and amount of catalyst. The observed results are summarized in Table 1.

Table 1. Screening of the amount of catalyst, solvents, and temperature in the one-pot synthesis of Hantzsch thiazole derivative 4 a.

\begin{tabular}{cccccc}
\hline Entry & Solvent & Catalysis (\%) & Time (h) & Temp ( $\left.{ }^{\circ} \mathbf{C}\right)$ & Yield (\%) \\
\hline 1 & Water & 15 & 24 & RT & 10 \\
2 & Water & 15 & 6 & 100 & 45 \\
3 & MeOH & 15 & 24 & RT & 18 \\
4 & MeOH & 15 & 4 & 60 & 55 \\
5 & EtOH & 15 & 24 & RT & 60 \\
6 & EtOH & 15 & 2 & 65 & 87 \\
7 & $1-B u t a n o l$ & 15 & 6 & 110 & 60 \\
8 & 2-propanol & 15 & 3 & 80 & 65 \\
9 & EtOH/Water & 15 & 24 & RT & 70 \\
10 & EtOH/Water & 15 & 2 & 65 & 87 \\
11 & EtOH/Water & - & 7 & 65 & 50 \\
12 & EtOH/Water & 5 & 2 & 65 & 74 \\
13 & EtOH/Water & 10 & 2 & 65 & 79 \\
14 & EtOH/Water & 18 & 2 & 65 & 87 \\
15 & EtOH/Water & 20 & 2 & 65 & 87 \\
\hline
\end{tabular}

Initially, we investigated the effect of various solvents in this model reaction. The above 3-(bromoacetyl)-4-hydroxy-6-methyl-2H-pyran-2-one (1), thiourea (2) and benzaldhyde (3a) were stirred in various solvents, such as water, ethanol, methanol, 1-butanol and 2-propanol at their respective reflux condition (Entries 2, 4, 6-8, Table 1). The reaction smoothly proceeded in 1-butanol, 2-propanol and water under reflux condition but not at ambient temperature $\left(25^{\circ} \mathrm{C}\right)$. These conditions remained unsuccessful for obtaining acceptable yields (Entries 1, 3, 5, Table 1), whereas the use of a mixture of ethanol/water $(50 / 50 \mathrm{v} / \mathrm{v})$ at reflux allowed the desired product in higher yield, typically from $50 \%$ to $87 \%$ (entries 10-16, Table 1). Therefore, the 1 to 1 mixture of ethanol/water represents the suitable, cheap, safe and environmentally benign solvent for the present investigation.

We further examined the amount of $\mathrm{SiW} . \mathrm{SiO}_{2}$ required for this reaction under the above conditions. The effect of catalyst amount on the multi-component reaction was investigated by varying the catalyst amount $(5 \%, 10 \%, 15 \%, 18 \%$ and $20 \%)$. It was found that when increasing the amount of the $\mathrm{SiW}_{\mathrm{SiO}}$ from $5 \%$ to $15 \%$, the yield also increased (Table 1). A further increase of the catalyst loading (above $15 \%$ ) does not affect the yield. Therefore, $15 \% \mathrm{SiW} \cdot \mathrm{SiO}_{2}$ in the mixture of ethanol/water is sufficient to push this reaction forward. Next, we investigated the reusability of $\mathrm{SiW}_{\mathrm{SiO}}$. At the end of the reaction, the catalyst could be recovered by a simple filtration. The recycled catalyst could be washed well with acetone and subjected to a second run of the reaction process. To ensure that the catalyst did not dissolve in acetone, it was weighted before using it then after filtration and reuse for the next reaction. The results show that this catalyst is not soluble in acetone. The catalyst remained active and exhibited no substantial loss of activity over up to three reaction cycles. The yield gradually decreased (72\%) for the fourth run. This may be due to loss of $\mathrm{SiW}$ on the $\mathrm{SiO}_{2}$ surface area.

Encouraged by the results obtained for the synthesis of $4 \mathbf{a}$, we extended this methodology to various substituted benzaldehydes $(\mathbf{3} \mathbf{b}-\mathbf{3} \mathbf{j})$, to prepare the corresponding thiazole derivatives $(\mathbf{4} \mathbf{b}-\mathbf{4} \mathbf{j})$ 
by two methods: under conventional heating (method A) and under ultrasonic irradiation (method B). By using ultrasonic activation assisted synthesis, at room temperature, the reaction time could be decreased compared to the previously reported conventional method as worked out for some analogs ( $2 \mathrm{~h}$ compared to $3.5 \mathrm{~h}$ ), without affecting the yield. The scope and generality of this process are illustrated with respect to various substituted benzaldehydes as shown in Table 2.

Table 2. Synthesis of Hantzsch thiazole derivatives under conventional heating and under ultrasonic irradiation.

\begin{tabular}{cccccccc}
\hline \multirow{2}{*}{ Product } & \multicolumn{3}{c}{ Substitutions } & \multicolumn{2}{c}{ Method A a } & \multicolumn{2}{c}{ Method B b } \\
\cline { 2 - 7 } & $\mathbf{R}_{\mathbf{1}}$ & $\mathbf{R}_{\mathbf{2}}$ & $\mathbf{R}_{\mathbf{3}}$ & Time (h) & Yield (\%) & Time (h) & Yield (\%) \\
\hline $\mathbf{4 a}$ & $\mathrm{H}$ & $\mathrm{H}$ & $\mathrm{H}$ & 2 & 87 & 1.5 & 90 \\
$\mathbf{4 b}$ & $\mathrm{H}$ & $\mathrm{OH}$ & $\mathrm{H}$ & 2 & 85 & 1.5 & 88 \\
$\mathbf{4} \mathbf{c}$ & $\mathrm{OH}$ & $\mathrm{H}$ & $\mathrm{OH}$ & 3.5 & 80 & 2 & 82 \\
$\mathbf{4 d}$ & $\mathrm{NO}_{2}$ & $\mathrm{H}$ & $\mathrm{H}$ & 2 & 82 & 1.5 & 85 \\
$\mathbf{4 e}$ & $\mathrm{Cl}$ & $\mathrm{H}$ & $\mathrm{H}$ & 2 & 84 & 1.5 & 87 \\
$\mathbf{4 f}$ & $\mathrm{OH}$ & $\mathrm{H}$ & $\mathrm{H}$ & 2 & 85 & 1.5 & 88 \\
$\mathbf{4 g}$ & $\mathrm{H}$ & $\mathrm{OH}$ & $\mathrm{OH}$ & 3.5 & 79 & 2 & 82 \\
$\mathbf{4 h}$ & $\mathrm{H}$ & $\mathrm{H}$ & $\mathrm{OCH}$ & 3.5 & 75 & 2 & 79 \\
$\mathbf{4 i}$ & $\mathrm{OCH}$ & $\mathrm{H}$ & $\mathrm{H}$ & 2 & 84 & 1.5 & 87 \\
$\mathbf{4 j}$ & $\mathrm{H}$ & $\mathrm{OCH}$ & $\mathrm{H}$ & 2 & 82 & 1.5 & 85 \\
\hline${ }^{\mathrm{a}}$ Conventional heating, $65{ }^{\circ} \mathrm{C}, \mathrm{ETOH} /$ Water $(50 / 50)$. & ${ }^{\mathrm{b}}$ Ultrasonic irradiation, RT, ETOH/Water (50/50).
\end{tabular}

In order to obtain more insight into the mechanism of the functionalized thiazoles formation, the stepwise reaction was studied. Thus, the intermediate (II) had been prepared by cyclocondensation of (1) with (2) in a mixture of ethanol and water $(50 / 50, v / v)$ under ultrasonic irradiation. The reaction of an equimolar amount of (II) with (3a) in the same conditions gave (4a) with a yield of $60 \%$. The characterisation of (II) had been reported in our previous work [50]. Our experiments showed that the target thiazole derivatives can be obtained "in one flask" by initially conducting cyclocondensation of the $\alpha$-haloketone (1) with thiourea (2) followed by condensation of the intermediaite (II)with substituted benzaldehydes (3a-3j) whichsupport the plausible mechanism as depicted in Scheme 2.

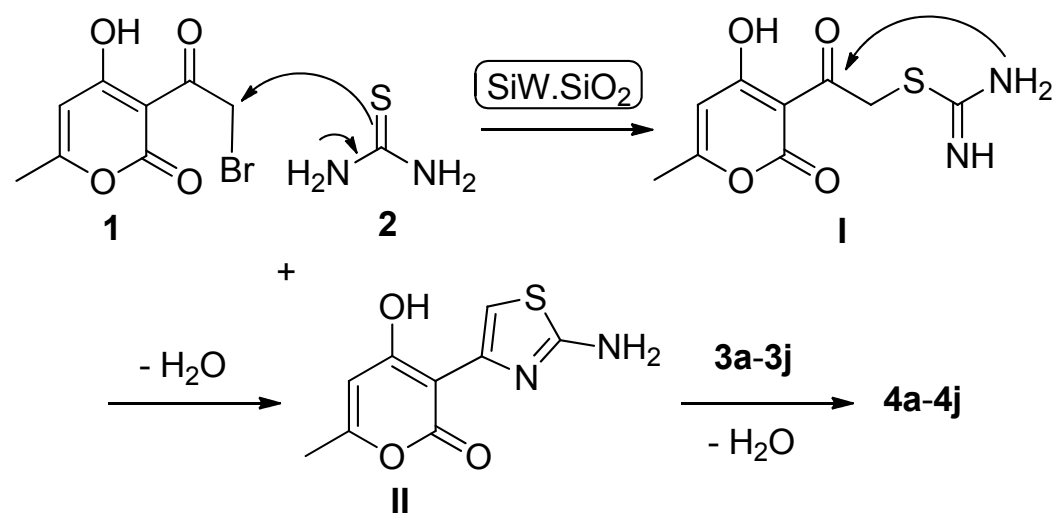

Scheme 2. A plausible mechanism for the formation of Hantzsch thiazole derivatives.

All the new target compounds were completely characterized by using infrared (IR), ${ }^{1} \mathrm{H}-\mathrm{NMR}$, ${ }^{13} \mathrm{C}-\mathrm{NMR}$ and mass spectroscopy (see supplementary). The spectroscopic data of the new compounds are given in the experimental section and are fully consistent with the proposed structures. IR spectra of $4 \mathbf{a}-4 \mathbf{j}$ had strong $\mathrm{N}=\mathrm{C}$ absorptions at about $1669 \mathrm{~cm}^{-1}$ and displayed absorptions at about 1617-1546 $\mathrm{cm}^{-1}$ and $1582 \mathrm{~cm}^{-1}$ which were assigned to $\mathrm{C}=\mathrm{O}$ and $\mathrm{C}=\mathrm{C}$ functionalities respectively. This study confirmed the conservation of 2-pyroneby the presence of large elongation and intense bands in the absorption range at $1718-1693 \mathrm{~cm}^{-1}$. The ${ }^{1} \mathrm{H}-\mathrm{NMR}$ spectra of the compounds $4 \mathbf{a}-4 \mathbf{j}$ exhibited broad 
signals at 6.02-6.92 ppm, which were assigned to the $\mathrm{C}-\mathrm{H}$ proton of the thiazole ring. ${ }^{13} \mathrm{C}-\mathrm{NMR}$ of compounds $4 \mathbf{a}-4 \mathbf{j}$ showed peaks at about 111.98-115.10 and 164.33-167.46 for C-S (thiazole) and C=N (amide), respectively.

\subsection{Biological Evaluation}

\subsubsection{Antioxidant Activity}

The 2,2-diphenyl-1-picrylhydrazil (DPPH) is a stable free radical that has been widely accepted as a tool for estimating the free radical scavenging activities of antioxidants [51]. The lower $\mathrm{IC}_{50}$ value indicates a stronger ability of the compound to act as a DPPH scavenger, while the higher $\mathrm{IC}_{50}$ value indicates a lower scavenging activity of the scavengers. This is because more scavengers were required to achieve a $50 \%$ scavenging reaction. In reaction with a hydrogen donor, the purple color of DPPH fades or disappears, due to its conversion to 2,2-diphenyl-1-picryl hydrazine, resulting in an absorbance decrease. The greater the decrease in absorption, in the presence of scavengers in the fractions, the more effective is its antioxidant activity.

In the current study, the antioxidant activity of compounds $(\mathbf{4 a}-\mathbf{4} \mathbf{j})$ was expressed as $\mathrm{IC}_{50}$ with a low $\mathrm{IC}_{50}$ value, indicating that the synthesis compounds act as an effective DPPH scavenger (Figure 1). $\mathrm{IC}_{50}$ values were between 11.23 and $16.96 \mu \mathrm{g} / \mathrm{mL}$ for the compounds $(\mathbf{4 a}-\mathbf{4 j})$, while $10.46 \mu \mathrm{g} / \mathrm{mL}$ for butylhydroxytoluene (BHT, positive control). The newly synthesized thiazoles display significant antioxidant capacitywith an insignificant variation, which indicates that the thiazole ring enhances the antioxidant activity of thiazoles, not the benzene ring where the variation is. However, compounds $\mathbf{4 b}$, $\mathbf{4} \mathbf{c}$ and $\mathbf{4} \mathbf{g}$ with both electrons donating and electron withdrawing groups in para position exhibited a promising antioxidant (DPPH) radical scavenging activity compared to BHT.

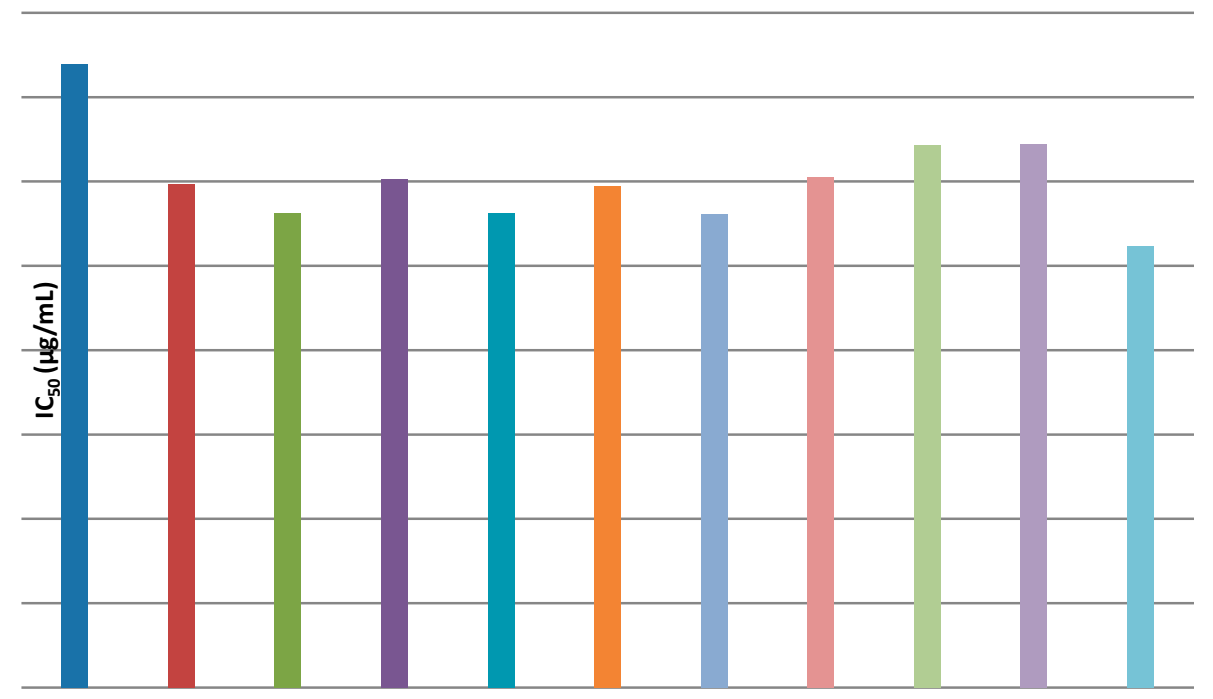

Figure 1. The antioxidant activity of compounds (4a-4j) using DPPH assay $\left(\mathrm{IC}_{50}=\mu \mathrm{g} / \mathrm{mL}\right)$, compared with reference standard butylhydroxytoluene (BHT) activity.

\subsubsection{In Vitro Antibacterial Activity}

The in vitro antibacterial activity of compounds $(\mathbf{4} \mathbf{a}-\mathbf{4 j})$, expressed as an MIC value, was assessed by the serial dilution methods against Escherichia coli, Staphylococcus aureus, Pseudomonas aeruginosa and two standard antibiotics (amoxicillin and ciprofloxacin) (Figure 2). The MICs of the synthesis compounds were within concentration ranges of 50-153 $\mu \mathrm{g} / \mathrm{mL}$. The entire synthesized compounds and standard antibiotics had higher antibacterial activity against bacteria strains and are most active against Gram-negative bacteria than the Gram-positive bacteria. In general, all compounds are, approximately, behaving as the reference compounds with same activities. This potent antibacterial 
activity exhibited may be due to the thiazole ring. However, we observed that both electron donating as well as electron withdrawing groups were found to increase the antibacterial properties. Compounds $4 \mathbf{b}, \mathbf{4 c}, \mathbf{4 f}$ and $\mathbf{4 g}$ displayed moderate antibacterial activity whereas the remaining compounds showed less activity.

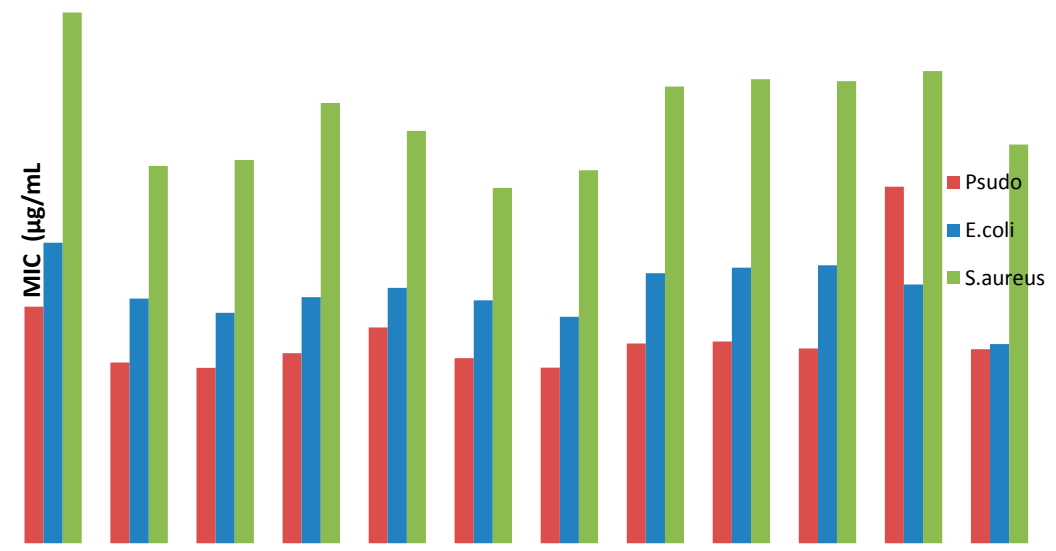

Figure 2. The antibacterial activity of compounds (4a-4j) compared to Ciprofloxacin and Amoxicillin $(\mathrm{MIC}=\mu \mathrm{g} / \mathrm{mL})$.

\section{Experimental Section}

\subsection{Materials and Methods}

All research chemicals and solvents were purchased from Sigma-Aldrich (mainly Sigma-Aldrich, Saint-Quentin-Fallavier, France) and were used as such for the reactions. The progress of all the reactions was monitored by thin-layer chromatography (TLC) using glass plates precoated with silica gel-60 F254 to a thickness of $0.5 \mathrm{~mm}$. The melting points were taken in an open capillary tube using Electrothermal melting point apparatus (Electrotermal, Rochford, Great Britain). The values are reported in ${ }^{\circ} \mathrm{C}$ and are uncorrected. IR spectra were recorded as neat solid or liquid on a Fourier Transform Bruker Vector 22 spectrometer (Villebon-sur-Yvette, France). NMR spectra were recorded with a Bruker Avance 300 spectrometer $\left[300 \mathrm{MHz}\left({ }^{1} \mathrm{H}\right)\right.$ and $\left.75 \mathrm{MHz}\left({ }^{13} \mathrm{C}\right)\right]$ (Bruker Biospin $\mathrm{GmbH}$, Rheinstetten, Germany). Chemical shifts are expressed in parts per million (ppm) downfield from Tetramethylsilane (TMS). Data are reported as follows: chemical shift [multiplicity (s: singlet, d: doublet, dd: double doublet, ddd: double double doublet, $\mathrm{dm}$ : double multiplet, $\mathrm{dt}$ : double triplet, $\mathrm{t}$ : triplet, $\mathrm{td}$ triple doublet, $\mathrm{tm}$, triple multiplet, $\mathrm{tt}$ : triple triplet, q: quartet, quint: quintuplet, $\mathrm{m}$ : multiplet, br: broad), coupling constants $(J)$ in Hertz, integration]. The numbers of attached proton(s) in the ${ }^{13} \mathrm{C}$-NMR spectra were elucidated by use of JMOD experiments (DEPT 135) and are described as $\left(\mathrm{CH}_{3}\right)$ methyl; $\left(\mathrm{CH}_{2}\right)$ methylene; $(\mathrm{CH})$ methine; $(\mathrm{C})$ quaternary carbon atoms. Shifts of ${ }^{1} \mathrm{H}$ - and ${ }^{13} \mathrm{C}-\mathrm{NMR}$ spectra were calibrated against the solvent residual isotopic peak as internal reference. Reference peaks for the NMR spectra in $\left(\mathrm{CD}_{3} \mathrm{~S}\right)_{2} \mathrm{O}: 2.50\left({ }^{1} \mathrm{H}\right)$ and $39.51\left({ }^{13} \mathrm{C}\right) \mathrm{ppm}$. Mass spectra were recorded on a Waters Micromass LCT Premier Q-TOF Mass spectrometer (Waters, Guyancourt, France) coupled with a Waters Alliance HPLC. Synthesis under ultrasonic irradiation was carried out with 3510E-MT Bransonic ultrasonic apparatus (Bransonic, Danbury, CT, USA).

\subsection{General Procedure for the Preparation of Compounds $(\mathbf{4 a}-\mathbf{4} \mathbf{j})$}

A mixture of 3-(bromoacetyl)-4-hydroxy-6-methyl-2H-pyran-2-one) ((1) (1 mmol), thiourea (2) (1 mmol), benzaldehyde (3a-3j) (1 mmol) and $\mathrm{SiW}_{\mathrm{SiO}}(15 \%)$ [36] was refluxed in ethanol/water 1/1 $(5 \mathrm{~mL})$ with stirring for $2 \mathrm{~h}$ to $3.5 \mathrm{~h}$ at $65^{\circ} \mathrm{C}$ or under ultrasonic activation for $1.5 \mathrm{~h}$ to $2 \mathrm{~h}$ at room temperature (Table 2).The obtained solid was filtered off and washed with ethanol; the remaining 
solid was dissolved in acetone and $\mathrm{SiW} \cdot \mathrm{SiO}_{2}$ was removed by filtration. The filtrated solution was evaporated under vacuum and the resulting product oven-dried $\left(60^{\circ} \mathrm{C}\right)$.

\subsection{Characterization Data of Synthesized Compounds}

4-Hydroxy-6-methyl-3-(2-[-phenylmethylidene]amino-1,3-thiazol-4-yl)-2H-pyran-2-one (4a). Yield 90\%; Yellow solid; m.p. $220^{\circ} \mathrm{C}$; IR neat $\left(\mathrm{cm}^{-1}\right)$ : 3050-3500, 2957, 2165, 1683, 1616, 1494, 1450, 1358, 1314, 1249, 1172, 1031, 997, 866, 827, 719. ${ }^{1} \mathrm{H}-\mathrm{NMR}\left(300 \mathrm{MHz},\left(\mathrm{CD}_{3} \mathrm{~S}\right){ }_{2} \mathrm{O}\right), \delta \mathrm{ppm}: 2.21\left(\mathrm{~s}, 3 \mathrm{H}, \mathrm{CH}_{3}\right)$, 5.52 (s, 1H, H-pyrone), 6.05 (s, 1H, H-thiazole), 7.25-7.72 (m, 5H, ArH), 7.93 (s, 1H, N=C-H), 9.16 (s, 1H, OH-pyrone); ${ }^{13} \mathrm{C}-\mathrm{NMR}\left(75 \mathrm{MHz},\left(\mathrm{CD}_{3} \mathrm{~S}\right)_{2} \mathrm{O}\right), \delta$ ppm: 19.68 ( $\mathrm{H}_{3} \mathrm{C}$-pyrone), 90.29 (C-3), 99.50 (C-5), 123.05 (C-S thiazole), 127.84, 128.20, 128.52, 128.63, 129.52, 130.77 (ArC and ArCH), 132.91 (C-N at thiazole), $138.61(\mathrm{C}-6), 161.59(\mathrm{C}=\mathrm{N}$ at thiazole), $164.54(\mathrm{C}-2), 167.35(\mathrm{C}=\mathrm{N}), 169.25(\mathrm{C}-4)$; HRMS (ESI) $m / z$ calc for $\mathrm{C}_{16} \mathrm{H}_{13} \mathrm{~N}_{2} \mathrm{O}_{3} \mathrm{~S} 313.0647$, found $313.0645[\mathrm{M}+\mathrm{H}]^{+}$.

4-Hydroxy-3-(2-[-(3-hydroxyphenyl)methylidene]amino-1,3-thiazol-4-yl)-6-methyl-2H-pyran-2-one (4b). Yield 88\%. Red solid; m.p. $128^{\circ} \mathrm{C}$; IR neat $\left(\mathrm{cm}^{-1}\right)$ : 3050-3500, 2919, 1934, 1681, 1617, 1491, 1450, 1360, 1310, 1210, 1170, 1036, 997, 873, 824, 722. ${ }^{1} \mathrm{H}-\mathrm{NMR}\left(300 \mathrm{MHz},\left(\mathrm{CD}_{3} \mathrm{~S}\right){ }_{2} \mathrm{O}\right), \delta$ ppm: $2.21\left(\mathrm{~s}, 3 \mathrm{H}, \mathrm{CH}_{3}\right), 5.47$ (s, 1H, H-pyrone), 6.05 (s, 1H, H-thiazole), $6.70(\mathrm{~d}, J=7.90 \mathrm{~Hz}, 1 \mathrm{H}, \mathrm{ArH}), 7.10(\mathrm{~d}, J=7.78 \mathrm{~Hz}, 1 \mathrm{H}, \mathrm{ArH})$, $7.25(\mathrm{~s}, 1 \mathrm{H}, \mathrm{ArH}), 7.36(\mathrm{~s}, 1 \mathrm{H}, \mathrm{N}=\mathrm{C}-\mathrm{H}), 7.44\left(\mathrm{t}, J_{1}=7.90 \mathrm{~Hz}, J_{2}=7.78 \mathrm{~Hz}, 1 \mathrm{H}, \mathrm{ArH}\right), 9.15(\mathrm{~s}, 1 \mathrm{H}, \mathrm{ArOH})$, $9.91\left(\mathrm{~s}, 1 \mathrm{H}, \mathrm{OH}-\right.$ pyrone); ${ }^{13} \mathrm{C}-\mathrm{NMR}\left(75 \mathrm{MHz},\left(\mathrm{CD}_{3} \mathrm{~S}\right){ }_{2} \mathrm{O}\right) \delta \mathrm{ppm}: 19.80\left(\mathrm{CH}_{3}\right.$-pyrone), 90.69 (C-3), 99.94 (C-5), 114.95(C-S at thiazole), 118.76, 121.53, 122.26, 123.60, 128.59 (ArC and ArCH), 129.94 (C-N at thiazole), 130.30 (ArC-OH), $140.53(\mathrm{C}-2), 157.69(\mathrm{C}=\mathrm{N}$ thiazole), $164.46(\mathrm{C}-6), 167.46(\mathrm{C}=\mathrm{N}), 169.66(\mathrm{C}-4)$; HRMS (ESI) $m / z$ calc for $\mathrm{C}_{16} \mathrm{H}_{13} \mathrm{~N}_{2} \mathrm{O}_{4} \mathrm{~S} 329.0596$, found $329.0597[\mathrm{M}+\mathrm{H}]^{+}$.

3-(2-[(2,4-Dihydroxyphenyl)methylidene]amino-1,3-thiazol-4-yl)-4-hydroxy-6-methyl-2H-pyran-2-one (4c). Yield $82 \%$. Brown solid; m.p. $237^{\circ} \mathrm{C}$; IR neat $\left(\mathrm{cm}^{-1}\right)$ : 3050-3500, 2956, 1718, 1681, 1613, 1503, 1450, 1358, 1284, 1209, 1167, 1036, 996, 862, 831, 716. ${ }^{1} \mathrm{H}-\mathrm{NMR}\left(300 \mathrm{MHz},\left(\mathrm{CD}_{3} \mathrm{~S}\right)_{2} \mathrm{O}\right), \delta$ ppm: $2.20\left(\mathrm{~s}, 3 \mathrm{H}, \mathrm{CH}_{3}\right)$, 5.43 (s, 1H, H-pyrone), 6.04 (s, 1H, H-thiazole), 6.44 (s, 1H, ArH), 6.65 (d, J = $7.88 \mathrm{~Hz}, 1 \mathrm{H}, \mathrm{ArH}), 6.79$ (d, $J=7.88 \mathrm{~Hz}, \mathrm{H}, \mathrm{ArH}), 7.09$ (s, 1H, N=C-H), 9.05 (s, 1H, ArOH), 10.18 (s, 1H, OH-pyrone); ${ }^{13} \mathrm{C}-\mathrm{NMR}$ (75 MHz, $\left.\left(\mathrm{CD}_{3} \mathrm{~S}\right)_{2} \mathrm{O}\right) \delta$ ppm: $19.52\left(\mathrm{CH}_{3}\right.$-pyrone), 37.81 (C-3), 90.16 (C-5), 99.73, 114.89, 118.31, 120.00 (ArC and $\mathrm{ArCH}), 123.32$ (C-S thiazole), 125.19 (C-N thiazole), 127.30 (C-2), 142.78, 145.04 (ArC-OH), 161.58 (C-6), 164.02 (C=N thiazole), $167.29(\mathrm{C}=\mathrm{N}), 169.25$ (C-4); HRMS (ESI) $m / z$ calc for $\mathrm{C}_{16} \mathrm{H}_{13} \mathrm{~N}_{2} \mathrm{O}_{5} \mathrm{~S}$ 345.0545, found $345.0552[\mathrm{M}+\mathrm{H}]^{+}$.

4-Hydroxy-6-methyl-3-(2-[(4-nitrophenyl)methylidene]amino-1,3-thiazol-4-yl)-2H-pyran-2-one (4d). Yield 85\%. Brown solid; m.p. $94{ }^{\circ} \mathrm{C}$; IR neat $\left(\mathrm{cm}^{-1}\right)$ : 3050-3500, 2957, 2174, 1684, 1608, 1563, 1510, 1450, 1358, $1303,1250,1179,1027,996,874,827,721 .{ }^{1} \mathrm{H}-\mathrm{NMR}\left(300 \mathrm{MHz},\left(\mathrm{CD}_{3} \mathrm{~S}\right)_{2} \mathrm{O}\right), \delta \mathrm{ppm}: 2.21\left(\mathrm{~s}, 3 \mathrm{H}, \mathrm{CH}_{3}\right)$, 6.05 (s, 1H, H-thiazole), 7.60 (s, 1H, H-pyrone), $8.15(\mathrm{~d}, J=8.51 \mathrm{~Hz}, 2 \mathrm{H}, \mathrm{ArH}), 8.40(\mathrm{~d}, J=8.51 \mathrm{~Hz}$, 2H, ArH), 9.21(s, 1H, OH-pyrone), 10.17 (s, 1H, N=C-H), ${ }^{13} \mathrm{C}-\mathrm{NMR}\left(75 \mathrm{MHz},\left(\mathrm{CD}_{3} \mathrm{~S}\right)_{2} \mathrm{O}\right), \delta$ ppm: 19.61 ( $\mathrm{H}_{3} \mathrm{C}$-pyrone), 90.65 (C-3) 99.42 (C-5), 121.43 (C-S at thiazole), 123.35, 123.81, 130.61, 136.04, 142.80 (ArC and $\mathrm{ArCH}), 150.54$ (C-N at thiazole), $161.60(\mathrm{C}-6), 164.61\left(\mathrm{ArC}-\mathrm{NO}_{2}\right), 167.45(\mathrm{C}=\mathrm{N}$ at thiazole), 167.70 (C-2), $171.43(\mathrm{C}=\mathrm{N}), 192.38$ (C-4);HRMS (ESI) $\mathrm{m} / \mathrm{z}$ calc for $\mathrm{C}_{16} \mathrm{H}_{12} \mathrm{~N}_{3} \mathrm{O}_{5} \mathrm{~S} 358.0498$, found 358.0495 $[\mathrm{M}+\mathrm{H}]^{+}$.

3-(2-[(4-Chlorophenyl)methylidene]amino-1,3-thiazol-4-yl)-4-hydroxy-6-methyl-2H-pyran-2-one (4e). Yield 87\%. Orange solid; $\mathrm{mp} 238^{\circ} \mathrm{C}$; IR neat $\left(\mathrm{cm}^{-1}\right)$ : 3050-3500, 2955, 1992, 1693, 1618, 1489, 1450, 1354, 1322, 1207, 1172, 1038, 997, 865, 821, 779, 721. ${ }^{1} \mathrm{H}-\mathrm{NMR}\left(300 \mathrm{MHz},\left(\mathrm{CD}_{3} \mathrm{~S}\right)_{2} \mathrm{O}\right), \delta \mathrm{ppm}: 2.20\left(\mathrm{~s}, 3 \mathrm{H}, \mathrm{CH}_{3}\right)$, $5.43(\mathrm{~s}, 1 \mathrm{H}, \mathrm{H}$-pyrone), $6.03(\mathrm{~s}, 1 \mathrm{H}, \mathrm{H}$-thiazole), $6.47(\mathrm{~d}, J=8.63 \mathrm{~Hz}, 1 \mathrm{H}, \mathrm{ArH}), 6.68(\mathrm{~d}, J=8.63 \mathrm{~Hz}, 1 \mathrm{H}$, $\mathrm{ArH}), 7.79(\mathrm{~d}, J=8.57 \mathrm{~Hz}, 1 \mathrm{H}, \mathrm{ArH}), 7.07(\mathrm{~s}, 1 \mathrm{H}, \mathrm{N}=\mathrm{C}-\mathrm{H}), 7.12(\mathrm{~d}, J=8.57 \mathrm{~Hz}, 1 \mathrm{H}, \mathrm{ArH}), 9.01(\mathrm{~s}, 1 \mathrm{H}$, OH-pyrone); ${ }^{13} \mathrm{C}-\mathrm{NMR}\left(75 \mathrm{MHz},\left(\mathrm{CD}_{3} \mathrm{~S}\right)_{2} \mathrm{O}\right), \delta$ ppm:19.50 ( $\mathrm{H}_{3} \mathrm{C}$-pyrone), 90.55 (C-3), 99.55 (C-5), 121.51 (C-S at thiazole), 128.31, 129.11, 129.68 (ArC and ArCH), 131.10 (C-N at thiazole), 132.38 (C-6), 137.75 (ArC-Cl), 161.55 (C=N at thiazole), 164.31 (C-2), 167.35 (C=N), 169.24 (C-4); HRMS (ESI) $m / z$ calc for $\mathrm{C}_{16} \mathrm{H}_{12} \mathrm{ClN}_{2} \mathrm{O}_{3} \mathrm{~S}$ 347.0257, found $347.0256[\mathrm{M}+\mathrm{H}]^{+}$. 
4-Hydroxy-3-(2-[(4-hydroxyphenyl)methylidene]amino-1,3-thiazol-4-yl)-6-methyl-2H-pyran-2-one (4f). Yield 88\%. Orange solid; m.p. $129^{\circ} \mathrm{C}$; IR neat $\left(\mathrm{cm}^{-1}\right)$ : 3050-3500, 2957, 1717, 1683, 1621, 1512, 1450, 1359, 1285, 1213, 1175, 1037, 996, 859, 835, 723. ${ }^{1} \mathrm{H}-\mathrm{NMR}\left(300 \mathrm{MHz},\left(\mathrm{CD}_{3} \mathrm{~S}\right){ }_{2} \mathrm{O}\right), \delta$ ppm: $2.21\left(\mathrm{~s}, 3 \mathrm{H}, \mathrm{CH}_{3}\right), 5.41$ (s, 1H, H-pyrone), 6.06 (s, 1H, H-thiazole), 6.69 (d, J = 8.7 Hz, 1H, ArH), 6.92 (d, J = 8.4 Hz, 1H, ArH), 7.07 (s, 1H, N=C-H), 7.04 (d, J = 8.4 Hz, 1H, ArH), $7.74(\mathrm{~d}, J=8.7 \mathrm{~Hz}, 1 \mathrm{H}, \mathrm{ArH}), 9.17(\mathrm{~s}, 2 \mathrm{H}, \mathrm{ArOH})$, 9.79 (s, 1H, OH-pyrone); ${ }^{13} \mathrm{C}-\mathrm{NMR}\left(75 \mathrm{MHz},\left(\mathrm{CD}_{3} \mathrm{~S}\right)_{2} \mathrm{O}\right), \delta$ ppm: $19.50\left(\mathrm{H}_{3} \mathrm{C}\right.$-pyrone), 90.55 (C-3), 99.55 (C-5), 121.51 (C-S at thiazole), 128.31, 129.11, 129.68 (ArC and $\mathrm{ArCH}), 131.10$ (C-N at thiazole), 132.38 (C-6), 137.75 (ArC-Cl), 161.55 (C=N at thiazole), $164.31(\mathrm{C}-2), 167.35$ (C=N), 169.24 (C-4); HRMS (ESI) $m / z$ calc for $\mathrm{C}_{16} \mathrm{H}_{13} \mathrm{~N}_{2} \mathrm{O}_{4} \mathrm{~S} 329.0596$, found $329.0595[\mathrm{M}+\mathrm{H}]^{+}$.

3-(2-[(2,3-Dihydroxyphenyl)methylidene]amino-1,3-thiazol-4-yl)-4-hydroxy-6-methyl-2H-pyran-2-one (4g). Yield 82\%. Brown solid; m.p. $134{ }^{\circ} \mathrm{C}$; IR neat $\left(\mathrm{cm}^{-1}\right)$ : 3050-3500, 2958, 2836, 1719, 1683, 1621, 1510, 1450, 1358, 1303, 1215, 1179, 1023, 995, 858, 832, 721. ${ }^{1} \mathrm{H}-\mathrm{NMR}\left(300 \mathrm{MHz},\left(\mathrm{CD}_{3} \mathrm{~S}\right){ }_{2} \mathrm{O}\right), \delta \mathrm{ppm}: 2.21(\mathrm{~s}, 3 \mathrm{H}$, $\mathrm{CH}_{3}$ ), 5.41 (s, 1H, H-pyrone), 6.06 (s, 1H, H-thiazole), 6.69 (d, J = 8.75 Hz, 1H, ArH), 6.92 (d, J = 8.39 Hz, 1H, ArH), 7.07 (s, 1H, N=C-H), $7.04(\mathrm{~d}, J=8.39 \mathrm{~Hz}, 1 \mathrm{H}, \mathrm{ArH}), 7.74(\mathrm{~d}, J=8.75 \mathrm{~Hz}, 1 \mathrm{H}, \mathrm{ArH}), 9.17(\mathrm{~s}, 2 \mathrm{H}$, $\mathrm{ArOH}), 9.79$ (s, 1H, OH-pyrone); ${ }^{13} \mathrm{C}-\mathrm{NMR}\left(75 \mathrm{MHz},\left(\mathrm{CD}_{3} \mathrm{~S}\right)_{2} \mathrm{O}\right), \delta$ ppm: 19.33 ( $\mathrm{H}_{3} \mathrm{C}$-pyrone), 90.29 (C-3), 99.49 (C-5), 115.10 (C-S at thiazole), 116.08, 123.56, 127.75, 128.55, 128.70 (ArC and ArCH), 132.04 (C-N at thiazole), $156.74(\mathrm{C}-6), 161.35(\mathrm{ArC}-\mathrm{OH}), 163.27(\mathrm{C}=\mathrm{N}$ at thiazole $), 164.35(\mathrm{C}-2), 167.02(\mathrm{C}=\mathrm{N})$, 169.12 (C-4); HRMS (ESI) $m / z$ calc for $\mathrm{C}_{16} \mathrm{H}_{13} \mathrm{~N}_{2} \mathrm{O}_{5} \mathrm{~S} 345.0545$, found $345.0541[\mathrm{M}+\mathrm{H}]^{+}$.

4-Hydroxy-3-(2-[(2-methoxyphenyl)methylidene]amino-1,3-thiazol-4-yl)-6-methyl-2H-pyran-2-one (4h). Yield 79\%. Green solid; m.p. $242^{\circ} \mathrm{C}$; IR neat $\left(\mathrm{cm}^{-1}\right)$ : 3050-3500, 2957, 2838, 1717, 1682, 1610, 1510, 1451, 1358, 1303, 1208, 1163, 1028, 996, 856, 827, 721. ${ }^{1} \mathrm{H}-\mathrm{NMR}\left(300 \mathrm{MHz},\left(\mathrm{CD}_{3} \mathrm{~S}\right)_{2} \mathrm{O}\right), \delta$ ppm: $2.17\left(\mathrm{~s}, 3 \mathrm{H}, \mathrm{CH}_{3}\right)$, $3.72\left(\mathrm{~s}, 3 \mathrm{H}, \mathrm{OCH}_{3}\right), 5.42\left(\mathrm{~s}, 1 \mathrm{H}, \mathrm{H}\right.$-pyrone), $6.05\left(\mathrm{~s}, 1 \mathrm{H}, \mathrm{H}\right.$-thiazole), $6.79\left(\mathrm{t}, J_{1}=11.31 \mathrm{~Hz}, J_{2}=9.77 \mathrm{~Hz}\right.$ 1H, ArH), $6.92(\mathrm{~d}, J=11.31 \mathrm{~Hz}, 1 \mathrm{H}, \mathrm{ArH}), 7.09$ (s, 1H, N=C-H), $7.22(\mathrm{~d}, J=10.51 \mathrm{~Hz}, 1 \mathrm{H}, \mathrm{ArH}), 7.67$ (t, $\left.J_{1}=11.31 \mathrm{~Hz}, J_{2}=9.77 \mathrm{~Hz} 1 \mathrm{H}, \mathrm{ArH}\right), 7.87(\mathrm{~s}, 1 \mathrm{H}, \mathrm{N}=\mathrm{C}-\mathrm{H}), 9.86$ (s, 1H, OH-pyrone); ${ }^{13} \mathrm{C}-\mathrm{NMR}(75 \mathrm{MHz}$, $\left.\left(\mathrm{CD}_{3} \mathrm{~S}\right)_{2} \mathrm{O}\right), \delta$ ppm: $19.46\left(\mathrm{H}_{3} \mathrm{C}\right.$-pyrone $), 40.33\left(\mathrm{OCH}_{3}\right), 55.16\left(\mathrm{ArC}-\mathrm{OCH}_{3}\right), 99.77(\mathrm{C}-3), 113.67$ (C-5), 114.56 (C-S at thiazole), 115.66, 123.75, 129.01 (ArC and $\mathrm{ArCH}), 129.45(\mathrm{C}-\mathrm{N}$ at thiazole), $158.55(\mathrm{C}=\mathrm{N}$ at thiazole), 161.63 (C-2), 163.91 (C-6), 167.17 (C=N), 169.25 (C-4); HRMS (ESI) $m / z$ calc for $\mathrm{C}_{17} \mathrm{H}_{15} \mathrm{~N}_{2} \mathrm{O}_{4} \mathrm{~S}$ 343.0753, found $343.0748[\mathrm{M}+\mathrm{H}]^{+}$.

4-Hydroxy-3-(2-[(4-methoxyphenyl)methylidene]amino-1,3-thiazol-4-yl)-6-methyl-2H-pyran-2-one (4i). Yield 87\%. Yellow solid; m.p. $204{ }^{\circ} \mathrm{C}$; IR neat $\left(\mathrm{cm}^{-1}\right)$ : 3050-3500, 2956, 1718, 1683, 1620, 1489, 1450, 1358, $1264,1210,1169,1034,995,870,822,721 .{ }^{1} \mathrm{H}-\mathrm{NMR}\left(300 \mathrm{MHz},\left(\mathrm{CD}_{3} \mathrm{~S}\right){ }_{2} \mathrm{O}\right), \delta$ ppm: $2.20\left(\mathrm{~s}, 3 \mathrm{H}, \mathrm{CH}_{3}\right), 3.71$ (s, 3H, OCH 3 ), 5.46 (s, 1H, H-pyrone), 6.02 (s, 1H, H-thiazole), 6.70 (d, J = 11.31 Hz, 2H, ArH), 7.09 (s, $1 \mathrm{H}, \mathrm{N}=\mathrm{C}-\mathrm{H}), 7.17$ (d, J = $11.31 \mathrm{~Hz}, 2 \mathrm{H}, \mathrm{ArH}), 9.90$ (s, 1H, OH-pyrone); ${ }^{13} \mathrm{C}-\mathrm{NMR}\left(75 \mathrm{MHz},\left(\mathrm{CD}_{3} \mathrm{~S}\right){ }_{2} \mathrm{O}\right)$, $\delta$ ppm: $19.59\left(\mathrm{H}_{3} \mathrm{C}\right.$-pyrone), $55.17\left(\mathrm{OCH}_{3}\right), 62.80\left(\mathrm{ArC}-\mathrm{OCH}_{3}\right), 90.26(\mathrm{C}-3), 99.52$ (C-5), 112.95 (C-S at thiazole), 119.86, 122.87, 128.08, 129.75 (ArC and $\mathrm{ArCH}), 140.03(\mathrm{C}-\mathrm{N}$ at thiazole), $159.28(\mathrm{C}=\mathrm{N}$ at thiazole), 161.63 (C-2), 164.61 (C-6), 167.37 (C=N), 169.09 (C-4); HRMS (ESI) $m / z$ calc for $\mathrm{C}_{17} \mathrm{H}_{15} \mathrm{~N}_{2} \mathrm{O}_{4} \mathrm{~S}$ 343.0753, found $343.0746[\mathrm{M}+\mathrm{H}]^{+}$.

4-Hydroxy-3-(2-[(3-methoxyphenyl)methylidene]amino-1,3-thiazol-4-yl)-6-methyl-2H-pyran-2-one (4j). Yield 85\%. Orange solid; m.p. $227^{\circ} \mathrm{C}$; IR neat $\left(\mathrm{cm}^{-1}\right)$ : 3050-3500, 2956, 1718, 1683, 1620, 1489, 1450, 1358, 1264, 1210, 1169, 1034, 995, 870, 822, 721. $\left.{ }^{1} \mathrm{H}-\mathrm{NMR}\left(300 \mathrm{MHz},\left(\mathrm{CD}_{3} \mathrm{~S}\right){ }_{2} \mathrm{O}\right)\right), \delta$ ppm: $2.21\left(\mathrm{~s}, 3 \mathrm{H}, \mathrm{CH}_{3}\right), 3.71$ $\left(\mathrm{s}, 3 \mathrm{H}, \mathrm{OCH}_{3}\right), 5.48$ (s, 1H, H-pyrone), 6.07 (s, 1H, H-thiazole), 6.78 (s, 1H, ArH), $6.84(\mathrm{~d}, J=11.31 \mathrm{~Hz}$, $1 \mathrm{H}, \mathrm{ArH}), 6.87(\mathrm{~d}, J=10.51 \mathrm{~Hz}, 1 \mathrm{H}, \mathrm{ArH}), 7.23\left(\mathrm{t}, J_{1}=11.31 \mathrm{~Hz}, J_{2}=10.51 \mathrm{~Hz}, 1 \mathrm{H}, \mathrm{ArH}\right), 7.28(\mathrm{~s}, 1 \mathrm{H}$, $\mathrm{N}=\mathrm{C}-\mathrm{H}), 9.21$ (s, 1H, OH-pyrone); ${ }^{13} \mathrm{C}-\mathrm{NMR}\left(75 \mathrm{MHz},\left(\mathrm{CD}_{3} \mathrm{~S}\right)_{2} \mathrm{O}\right), \delta$ ppm: $19.51\left(\mathrm{H}_{3} \mathrm{C}\right.$-pyrone $), 55.77$ $\left(\mathrm{OCH}_{3}\right), 90.26$ (C-3), 99.52 (C-5), 112.53(C-S at thiazole), 113.98, 120.08, 122.87, 128.16, 129.42 (ArC and $\mathrm{ArCH}), 140.03$ (C-N at thiazole), $158.81\left(\mathrm{C}=\mathrm{N}\right.$ and $\left.\mathrm{ArC}-\mathrm{OCH}_{3}\right), 161.37(\mathrm{C}=\mathrm{N}$ at thiazole), $164.45(\mathrm{C}-2)$, 167.29 (C-6), 169.18 (C-4); HRMS $m / z$ calc for $\mathrm{C}_{17} \mathrm{H}_{15} \mathrm{~N}_{2} \mathrm{O}_{4} \mathrm{~S} 343.0753$, found $343.0753[\mathrm{M}+\mathrm{H}]^{+}$. 


\section{Pharmacological Assay}

\subsection{Antioxidant Activity Evaluation}

The antioxidant capacity of compounds $\mathbf{4 a}-\mathbf{4 k}$ was evaluated by the method of Wang et al. 52(1998) [52]. The absorbance was measured at $517 \mathrm{~nm}$ against a blank, i.e., without DPPH. All tests were run in triplicate and an average was used. Decrease of DPPH solution absorbance indicates an increase of DPPH radical scavenging activity. The amount of sample necessary to decrease the absorbance of DPPH by 50\% (IC50) was calculated graphically and the percentage inhibition was calculated according to the equation:

$$
\% \text { inhibition }=\frac{(\text { Ao }- \text { At })}{\text { Ao }} \times 100
$$

where $A_{o}$ is the absorbance of the control at $t=0 \mathrm{~min}$; and $A_{t}$ is the absorbance of the antioxidant at $\mathrm{t}=30 \mathrm{~min}$. The food preservative butylhydroxytoluene (BHT) was used as positive control.

\subsection{Antibacterial Assay}

Clinical isolation of two Gram-negative (Escherichia coli ATCC 25922 and Pseudomonas aeruginosa ATCC 27853) and one Gram-positive (Staphylococcus aureus ATCC 43300) bacteria strains was obtained from the Laboratory of Cellular and Molecular Biology, Faculty of Biological Sciences, Houari Boumediene University of Sciences and Technology (USTHB), Algeria. The minimum inhibitory concentration (MIC) values were evaluated using the serial dilution method according to standard methods (NCCLS, 532003) [53]. Stock solutions of the compounds $\mathbf{4 a - 4 k}$ and antibiotic standards (amoxicillin and ciprofloxacin) were prepared in DMSO. Dilution series, using MHB, were prepared from 10 to $200 \mu \mathrm{g} / \mathrm{mL}$. After incubation at $37^{\circ} \mathrm{C}$ for $24 \mathrm{~h}$, the microorganism growth inhibition was evaluated by measuring absorbance at $630 \mathrm{~nm}$, using a spectrophotometer. Experiments were performed in triplicate at three different times.

\section{Conclusions}

In conclusion, an efficient and simple one-pot multi-component condensation procedure was introduced and developed for the synthesis of new thiazole derivatives, under conventional heating or under ultrasound irradiation, using $\mathrm{SiW}_{\mathrm{SiO}}$ as a reusable catalyst. The present method is bestowed with several advantages, such as an inexpensive and efficient catalyst, high reaction rate, high yield, simple workup procedure and high regioselectivity. Utilizing ultrasonic irradiation techniques provided dramatic improvements in terms of higher yields and shorter reaction times compared with the conventional heating method. This procedure would be a valuable addition to the current methodologies.

The preliminary studies of these compounds proved that the thiazole ring enhances the antibacterial as well asantioxidantactivities of the synthesis thiazoles, which might serve as new templates in the synthesis and development of potent therapeutics.

Supplementary Materials: Supplementary materials are available online.

Acknowledgments: The authors wish to thank Karine Leblanc (Faculty of Pharmacy, Châtenay-Malabry, France) for high resolution mass spectra. This work was supported by The Ministry of Higher Education and Scientific Research (Algeria), the National Center of the scientific research, and the Ministry of Education, Research, and Technology (France).

Author Contributions: Yahia Rachedi and Houria Bouherrou conceived and designed the experiments; Houria Bouherrou and Aicha Saidoun performed the experiments; Yahia Rachedi and Houria Bouherrou analyzed the data; Françoise Dumasand Albert Demenceau contributed analysis tools; Ahmed Abderrahmani and Lamia Abdellaziz realised biological evaluation; Yahia Rachedi wrote the paper.

Conflicts of Interest: The authors declare no conflict of interest. 


\section{References}

1. Yu, Y.B.; Chen, H.L.; Wang, L.Y.; Chen, X.L.; Fu, B. A facile synthesis of 2,4-disubstituted thiazoles using $\mathrm{MnO}_{2}$. Molecules 2009, 14, 4858-4865. [CrossRef] [PubMed]

2. Dawane, B.S.; Konda, S.G.; Kamble, V.T.; Chavan, S.A.; Bhosale, R.B.; Baseer, S. Multicomponent one-pot synthesis of substituted Hantzsch thiazole derivatives under solvent free conditions. E-J. Chem. 2009, 6, 358-362. [CrossRef]

3. Sharshira, E.M.; Hamada, N.M.M. Synthesis, characterization and antimicrobial activities of some thiazole derivatives. Am. J. Org. Chem. 2012, 2, 69-73. [CrossRef]

4. Sadjadikhah, S.S.; Maghsoodlou, M.T.; Hazeri, N.; Khorassani, S.M.; Najafi, S.G. One-Pot multicomponent synthesis of rightly substituted piperidines using p-toluene sulfonic acid monohydrate as catalyst. Monatsh. Chem. 2012, 143, 939-943. [CrossRef]

5. Andreani, A.; Rambaldi, M.; Leoni, A.; Locatelli, A.; Bossa, R.; Chiericozzi, M.; Galatulas, I.; Salvatore, G. Synthesis and cardiotonic activity of imidazo[2,1-b]thiazoles bearing a lactam ring. Eur. J. Med. Chem. 1996, 31, 383-387. [CrossRef]

6. Aggarwal, R.; Kumar, S.; Kaushik, P.; Kaushik, D.; Gupta, G.K. Synthesis and pharmacological evaluation of some novel 2-(5-hydroxy-5-trifluoromethyl-4,5-dihydropyrazol-1-yl)-4-(coumarin-3-yl)thiazoles. Eur. J. Med. Chem. 2013, 62, 508-514. [CrossRef] [PubMed]

7. Saeed, S.; Rashid, N.; Jones, P.G.; Hussain, R.; Bhatti, M.H. Spectroscopic characterization, crystal structure and antifungal activity of thiourea derivatives containing a thiazole moiety. Cent. Eur. J. Chem. 2010, 8, 550-558. [CrossRef]

8. Liu, H.L.; Li, Z.; Anthonsen, T. Synthesis and fungicidal activity of 2-imino-3-(4-arylthiazol-2-yl)thiazolidin-4-ones and their 5-arylidene derivatives. Molecules 2000, 5, 1055-1061. [CrossRef]

9. Argyropoulou, I.; Geronikaki, A.; Vicini, P.; Zani, F. Synthesis and biological evaluation of sulfonamide thiazole and benzothiazole derivatives as antimicrobial agents. Arkivoc 2009, 6, 89-102.

10. Amine, M.A.K.; Abdel Rahman, D.E.; El-Eryani, Y.A. Synthesis and preliminary evaluation of some substituted coumarins as anticonvulsant agents. Bioorg. Med. Chem. 2008, 16, 5377-5388. [CrossRef] [PubMed]

11. Chowki, C.S.; Magdum, P.L.; Ladda, S.K. Synthesis and antitubercular activity of 6-nitro-2-[4-formyl-3(substituted phenyl)pyrazol-1-yl]benzothiazoles. Int. J. Chem. Sci. 2008, 6, 1600-1605.

12. Karegoudar, P.; Karthikeyan, M.S.; Prasad, D.J.; Mahalinga, M.; Holla, B.S.; Kumari, N.S. Synthesis of some novel 2,4-disubstituted thiazoles as possible antimicrobial agents. Eur. J. Med. Chem. 2008, 43, 261-267. [CrossRef] [PubMed]

13. Ali, E.-S.T.; Kazak, A.M. Synthesis and antimicrobial activity of some new 1,3-thiazoles,1,3,4-thiadiazoles, 1,2,4-triazoles and 1,3-thiazines incorporating acridine and 1,2,3,4-tetrahydroacridine moieties. Eur. J. Chem. 2010, 1, 6-11. [CrossRef]

14. Badiger, N.P.; Khan, A.; Kalashetti, M.B.; Khazi, I.M. Synthesis and local anaesthetic activities of 2-aminothiazole/thiadiazole analogues of lidocaine. Med. Chem. Res. 2012, 21, 1544-1549. [CrossRef]

15. Hutchinson, I.; Jennings, S.A.; Vishnuvajjala, B.R.; Westwell, A.D.; Stevens, M.F.G. Synthesis and pharmaceutical properties of antitumor 2-(4-aminophenyl)benzothiazole amino acid prodrugs. J. Med. Chem. 2002, 45, 744-747. [CrossRef] [PubMed]

16. Azarifar, D.; Shaebanzadeh, M. Synthesis and characterization of new 3,5-dinaphthyl substituted 2-pyrazolines and study of their antimicrobial activity. Molecules 2002, 7, 885-895. [CrossRef]

17. Potewar, T.M.; Ingale, S.A.; Srinivasan, K.V. Catalyst-Free efficient synthesis of 2-aminothiazoles in water at ambient temperature. Tetrahedron 2008, 64, 5019-5022. [CrossRef]

18. Kabalka, G.W.; Mereddy, A.R. Microwave promoted synthesis of functionalized 2-aminothiazoles. Tetrahedron Lett. 2006, 47, 5171-5172. [CrossRef]

19. Ding, Q.; Zhu, D.; Jin, H.; Chen, J.; Ding, J.; Wu, H. Eco-Friendly one-pot synthesis of 2,4-disubstituted thiazoles by grinding under catalyst- and solvent-free conditions. Phosphorus Sulfur Silicon Relat. Elem. 2011, 186, 220-224. [CrossRef]

20. Potewar, T.M.; Ingale, S.A.; Srinivasan, K.V. Efficient synthesis of 2,4-disubstituted thiazoles using ionic liquid under ambient conditions: A practical approach towards the synthesis of fanetizole. Tetrahedron 2007, 63, 11066-11069. [CrossRef] 
21. Penta, S.; Gadidasu, K.K.; Basavoju, S.; Rao, V.R. An efficient one-pot synthesis of pyrazolyl-[1,2,4]triazolo $[3,4-b][1,3,4]$ thiadiazin-6-yl)-2H-pyran-2-one derivatives via multicomponent approach and their potential antimicrobial and nematicidal activities. Tetrahedron Lett. 2013, 54, 5663-5666. [CrossRef]

22. Rajendran, A.; karthikeyan, C.; Rajathi, K.; Ragupathy, D. An environmentally benign one pot synthesis of substituted quinolines catalysed by fluoroboric acid based ionic liquid. J. Chem. Sci. 2012, 124, 877-881. [CrossRef]

23. Aoyama, T.; Murata, S.; Arai, I.; Araki, N.; Takido, T.; Suzuki, Y.; Kodomari, M. One-Pot synthesis using supported reagents system $\mathrm{KSCN} / \mathrm{SiO}_{2}-\mathrm{RNH}_{3} \mathrm{OAc} / \mathrm{Al}_{2} \mathrm{O}_{3}$ : Synthesis of 2-aminothiazoles and $\mathrm{N}$-allylthioureas. Tetrahedron 2006, 62, 3201-3213. [CrossRef]

24. Jing, X.; Li, Z.; Pan, X.; Shi, Y.; Yan, C. NaIO ${ }_{4}$-Catalysed one-pot synthesis of dihydropyrimidinones at room temperature under solvent-free conditions. J. Iran. Chem. Soc. 2009, 6, 514-518. [CrossRef]

25. Narender, M.; Reddy, M.S.; Sridhar, R.; Nageswara, Y.V.D.; Rao, K.R. Aqueous phase synthesis of thiazoles and aminothiazoles in the presence of $\beta$-cyclodextrin. Tetrahedron Lett. 2005, 46, 5953-5955. [CrossRef]

26. Bai, N.; Sha, Y.W.; Meng, G. Efficient and eco-friendly preparation of 4-methyl-5-formylthiazole. Molecules 2008, 13, 943-947. [CrossRef] [PubMed]

27. Penta, S.; Vedula, R.R. Environmentally friendly one-pot synthesis of substituted thiazoles and thiazolylpyrazoles. Russ. J. Gen. Chem. 2012, 82, 1403-1406. [CrossRef]

28. Ramachandran, R.; Jayanthi, S.; Jeong, Y.T. One-Pot synthesis of highly diversified tetrahydropyridines by tandem condensation of aldehydes, amines, and $\beta$-ketoesters. Tetrahedron 2012, 68, 363-369. [CrossRef]

29. Sapi, J.; Laronze, J.Y. Indole based multicomponent reactions towards functionalized heterocycles. Arkivoc 2004, 7, 208-222. [CrossRef]

30. Litvinov, Y.M.; Rodinovskaya, L.A.; Shestopalov, A.M. A new convenient four-component synthesis of 6-amino-2H,4H-pyrano [2,3-c]pyrazole-5-carbonitriles and one-pot synthesis of $6^{\prime}$-amino-5'-cyano-1,2dihydrospiro-[(3H)-indole-3, $4^{\prime}-\left(4^{\prime} H\right)$ pyrano[2,3-c]pyrazol]-2-ones. Russ. Chem. Bull. 2009, 58, 2362-2368. [CrossRef]

31. Shahriza, W.A.; Esmati, S.; Nazari, N.G. Boric acid as mild and efficient catalyst for one-pot synthesis of 1-amidoalkyl-2-naphtols under solvent-free conditions. J. Chem. Sci. 2012, 124, 927-931. [CrossRef]

32. Wang, Y.; Chen, M.; Ding, M.W. A simple and one-pot synthesis of 2,3,4,5-tetrasubstituted 4,5-dihydro-3H-1,4-benzodiazepines. Tetrahedron 2013, 69, 9056-9062. [CrossRef]

33. Santhosh1, P.; Chunduru1, V.S.R.; Rao, V.R. One-Pot synthesis of trisubstituted pyrazoles via multicomponent approach. Chem. Heterocycl. Compd. 2011, 47, 547-551. [CrossRef]

34. Thirupaiah, B.; Vedula, R.R. Novel one-pot multicomponent synthesis of substituted 2,3-dihydro-2-(6(4-hydroxy-6-methyl-2-oxo-2H-pyran-3-yl)-7H-[1,2,4]triazolo[3,4-b][1,3,4]thiadiazin-3-yl)phthalazine-1,4diones and substituted 3-[3-(N'-benzylidene-hydrazino)-7H-[1,2,4]Triazolo[3,4- $b][1,3,4]$ thiadiazin-6-yl]-4hydroxy-6-methyl-pyran-2-ones. Synth. Commun. 2014, 44, 513-519.

35. Morin, P.; Hamad, B.; Sapaly, G.; Carneiro Rocha, M.G.; Pries de Oliveira, P.G.; Gonzalez, W.A.; Andrade Sales, E.; Essayem, N. Transesterification of rapeseed oil with ethanol: I. Catalysis with homogeneous Kegginheteropolyacids. Appl. Catal. A 2007, 330, 69-76. [CrossRef]

36. Caetano, C.S.; Fonseca, I.M.; Ramos, A.M.; Vital, J.; Castanheiro, J.E. Esterification of free fatty acids with methanol using heteropolyacids immobilized on silica. Catal. Commun. 2008, 9, 1996-1999. [CrossRef]

37. Zieba, A.; Matachowski, L.; Gurgul, A.; Bielañska, E.; Drelinkiewicz, A. Transesterification reaction of triglycerides in the presence of Ag-doped $\mathrm{H}_{3} \mathrm{PW}_{12} \mathrm{O}_{40}$. J. Mol. Catal. A Chem. 2010, 316, 30-44. [CrossRef]

38. Ferreira, P.; Fonseca, I.M.; Ramos, A.M.; Vital, J.; Castanheiro, J.E. Glycerol acetylation over dodecatungstophosphoric acid immobilized into a silica matrix as catalyst. Appl. Catal. B 2009, 91, 416-422. [CrossRef]

39. Ferreira, P.; Fonseca, I.M.; Ramos, A.M.; Vital, J.; Castanheiro, J.E. Acetylation of glycerol over heteropolyacids supported on activated carbon. Catal. Commun. 2011, 12, 573-576. [CrossRef]

40. Yadav, J.S.; Reddy, B.V.S.; Reddy, K.S. Ultrasound-Accelerated synthesis of chiral allylic alcohols promoted by indium metal. Tetrahedron 2003, 59, 5333-5336. [CrossRef]

41. Gao, D.M.; Ma, W.L.; Li, T.R.; Huang, L.Z.; Du, Z.T. An improved synthesis of 1,2-diarylethanols under conventional heating and ultrasound irradiation. Molecules 2012, 17, 10708-10715. [CrossRef] [PubMed]

42. Disselkamp, R.S.; Hart, T.R.; Williams, A.M.; White, J.F.; Peden, C.H.F. Ultrasound-Assisted hydrogenation of cinnamaldehyde. Ultrason. Sonochem. 2005, 12, 319-324. [CrossRef] [PubMed] 
43. Lv, G.S.; Junduan, F.; Ding, J.C.; Cheng, T.X.; Gao, W.X.; Chen, J.X.; Wu, H.Y. Tandem base-free synthesis of $\beta$-hydroxy sulphides under ultrasound irradiation. J. Chem. Sci. 2012, 124, 1057-1062. [CrossRef]

44. Singh, A.K.; Shukla, S.K.; Quraishi, M.A. Ultrasound mediated green synthesis of hexa-hydro triazines. J. Mater. Environ. Sci. 2012, 2, 403-406.

45. Ding, L.; Wang, W.; Zhang, A. Synthesis of 1,5-dinitroaryl-1,4-pentadien-3-ones under ultrasound irradiation. Ultrason. Sonochem. 2007, 14, 563-567. [CrossRef] [PubMed]

46. Soltani, M.N. Ultrasound promoted mild and facile one-pot, three component synthesis of $2 H$-indazoles by consecutive condensation, C-N and N-N bond formations catalysed by copper-doped silica cuprous sulphate (CDSCS) as an efficient heterogeneous nano-catalyst. Ultrason. Sonochem. 2017, 34, 865-872. [CrossRef] [PubMed]

47. Hikem-Oukacha, D.; Hamdi, M.; Silva, A.M.S.; Rachedi, Y. New synthesis and reactivity of 3-bromoacetyl-4hydroxy-6-methyl-2H-pyran-2-one with binucleophilic amines. J. Heterocycl. Chem. 2011, 48, 63-68. [CrossRef]

48. Boswell, G.E.; Musso, D.L.; Kelley, J.L.; Soroko, F.E.; Cooper, B.R. Synthesis and anti-tetrabenazine activity of C-3 analogues of dimethyl-2-phenylmorpholines. J. Heterocycl. Chem. 1996, 33, 33-39. [CrossRef]

49. Lepeshkin, A.Y.; Turchin, K.F.; Gal'pern, E.G.; Stankevich, I.V.; Lyssenko, K.A.; Velezheva, V.S. Mechanism of dehydration of 2-CH2R- and 2- $\mathrm{CHR}_{2-4}$-hydroxy- $\Delta^{2}$-thiazolines as intermediates in the hantzsch thiazole synthesis and factors impeding the synthesis of 2-Me-, 2-Ar-, and -Het-substituted thiazoles and thiazolo[5,4- $b$ ]indoles. Russ. Chem. Bull. 2007, 56, 1447-1455. [CrossRef]

50. Hikem-Oukacha, D.; Hamdi, M.; Silva, A.M.S.; Rachedi, Y. Synthesis and reactivity of 6-methyl-4Hfuro[3,2c]pyran-3,4-dione. J. Heterocycl. Chem. 2011, 48, 31-37. [CrossRef]

51. Hu, F.L.; Lu, R.L.; Huang, B.; Ming, L. Free radical scavenging activity of extracts prepared from fresh leaves of selected Chinese medicinal plants. Fitoterapia 2004, 75, 14-23. [PubMed]

52. Wang, M.; Li, J.; Rangarajan, M.; Shao, Y.; La Voie, E.J.; Huang, C.T.; Ho, C.T. Antioxidative phenolic compounds from sage (Salvia officinalis). J. Agric. Food Chem. 1998, 46, 4869-4873. [CrossRef]

53. Abdollah, G.P.; Iraj, H.; Hamze, A.S. Essential oil variation, antioxidant and antibacterial activity of mountain fennel (Zaravschanicamembranacea (Boiss.) M. Pimen.). Ind. Crops Prod. 2013, 50, 443.

Sample Availability: Samples of the compounds $\mathbf{4 a}-\mathbf{4} \mathbf{j}$ are available from the authors.

(c) 2017 by the authors. Licensee MDPI, Basel, Switzerland. This article is an open access article distributed under the terms and conditions of the Creative Commons Attribution (CC BY) license (http:/ / creativecommons.org/licenses/by/4.0/). 